LA W RENCE LIVERM ORE NATIONAL LABORATORY

Electric Dipole Transitions Within The Ab initio No-Core Shell Model With Continuum

S. Quaglioni, S. Baroni, P. Navratil

October 8, 2012 
This document was prepared as an account of work sponsored by an agency of the United States government. Neither the United States government nor Lawrence Livermore National Security, LLC, nor any of their employees makes any warranty, expressed or implied, or assumes any legal liability or responsibility for the accuracy, completeness, or usefulness of any information, apparatus, product, or process disclosed, or represents that its use would not infringe privately owned rights. Reference herein to any specific commercial product, process, or service by trade name, trademark, manufacturer, or otherwise does not necessarily constitute or imply its endorsement, recommendation, or favoring by the United States government or Lawrence Livermore National Security, LLC. The views and opinions of authors expressed herein do not necessarily state or reflect those of the United States government or Lawrence Livermore National Security, LLC, and shall not be used for advertising or product endorsement purposes.

This work performed under the auspices of the U.S. Department of Energy by Lawrence Livermore National Laboratory under Contract DE-AC52-07NA27344. 


\title{
Electric dipole transitions within the ab initio no-core shell model with continuum
}

\author{
Sofia Quaglioni, ${ }^{1}$ Simone Baroni, ${ }^{2,3}$ and Petr Navrátil ${ }^{1,3}$ \\ ${ }^{1}$ Lawrence Livermore National Laboratory, P.O Box 808, L-414, Livermore, California 94551, USA \\ ${ }^{2}$ Physique Nucléaire Théorique, Université Libre de Bruxelles, C.P. 229, B-1050 Bruxelles, Belgium \\ ${ }^{3}$ TRIUMF, 4004 Wesbrook Mall, Vancouver BC, V6T 2A3, Canada
}

The no-core shell model with continuum (NCSMC) offers a unified approach to nuclear bound and continuum states based on the coupling of the no-core shell model (NCSM), a bound-state technique, with the no-core shell model/resonating group method (NCSM/RGM), a nuclear scattering technique. This new ab initio method leads to convergence properties superior to either NCSM or NCSM/RGM while providing a balanced approach to different classes of states. The NCSMC manynucleon wave function contains: $i$ ) a pre-diagonalized $A$-nucleon NCSM component expanded in the harmonic oscillator basis; ii) a binary-cluster NCSM/RGM component with asymptotic boundary conditions that properly describe weakly bound states, resonances and scattering. Here, we review the main aspects of the NCSM formalism, and provide algebraic expression for the reduced matrix elements of the electric dipole operator, needed to calculate E1 transition probabilities, radiative captures and photodisintegration cross sections. 


\section{INTRODUCTION}

In the recent past, significant effort has been devoted to extend ab initio techniques to the treatment of dynamical processes among light nuclei and arrive at the accurate prediction of low-energy nuclear reactions important for astrophysics and energy generation on earth, but also of the properties of light exotic nuclei for which most low-lying states are unbound, so that a rigorous analysis requires scattering boundary conditions.

To this aim, we combined the ab initio no-core shell model (NCSM) [1] and the resonating-group method (RGM) [27] techniques into a new many-body approach, ab initio NCSM/RGM [8, 9], capable of treating bound and scattering states of light nuclei in a unified formalism, starting from the fundamental inter-nucleon interactions. The NCSM/RGM approach has been successfully applied to a wide variety of processes, such as nucleon- ${ }^{4} \mathrm{He}$ and $n-{ }^{7} \mathrm{Li}$ scattering [10], ${ }^{7} \mathrm{Be}(p, \gamma){ }^{8} \mathrm{~B}$ capture [11], $d-{ }^{4} \mathrm{He}$ scattering [12], ${ }^{3} \mathrm{H}(d, n){ }^{4} \mathrm{He}$, and ${ }^{3} \mathrm{He}(d, p){ }^{4} \mathrm{He}$ fusion [13]. At the same time, these studies have highlighted practical limitations of the approach mainly related to a non-entirely efficient description of short-to-medium $A$-body correlations, that have to be treated by including a large number of excited states of the clusters.

The inefficiencies discussed above can be overcome working within an extended basis, in which the binary-cluster $(A-a, a)$ NCSM/RGM states are augmented by pre-diagonalized $A$-nucleon NCSM states. Indeed, the NCSM sector of the basis provides an effective description of the short- to medium-range A-body structure, while the NCSM/RGM cluster states make the theory able to handle the scattering physics of the system. In other words, one obtains the coupling of the NCSM with the continuum or NCSMC. This formalism was first mentioned in our review paper [14] and is now being successfully applied to the $a b$ initio description of the low-lying resonances of the exotic ${ }^{7}$ He nucleus [15]. We note that an analogous approach was already suggested in the original RGM papers $[2,3]$.

The NCSMC offers an optimal framework for the description of complex exotic nuclear structures, such as, e.g., those encountered among the Beryllium isotopes, grounded within the fundamental interactions among nucleons. Indeed, the Be isotopic chain exhibits an unusual structure evolution, ranging from the $\alpha$-cluster structure in ${ }^{8,9} \mathrm{Be}$ to the one-neutron halo structure in ${ }^{11} \mathrm{Be}$, in which short-, medium- and long-range correlations all play a non-negligible role. Of particular interest are the enhanced E1 transitions from the ground state to the first excited state in ${ }^{9,11}$ Be, which provide among the most sensitive tests of modern realistic interactions and calculated many-body wave functions, and have been recently re-measured at TRIUMF using the low-energy Coulomb excitation technique to improve the experimental accuracy to within $5 \%$ [16].

While the main components of the NCSMC approach have been introduced in Ref. [15], here we provide the algebraic expressions for the reduced matrix element between NCSMC basis states of the electric dipole operator. The fairly detailed overview of the NCSMC formalism presented in Sec. II supports the derivation of the E1 reduced matrix elements given in Sec. III. Conclusions and outlook are given in Sect. IV.

\section{NCSMC FORMALISM}

In the NCSMC, the wave function for a system of $A$ interacting nucleons is cast in the form

$$
\left|\Psi_{A}^{J^{\pi} T}\right\rangle=\sum_{\lambda} c_{\lambda}^{J^{\pi} T}\left|A \lambda J^{\pi} T\right\rangle+\sum_{\nu} \int d r r^{2} \frac{\gamma_{\nu}^{J^{\pi} T}(r)}{r} \hat{\mathcal{A}}_{\nu}\left|\Phi_{\nu r}^{J^{\pi} T}\right\rangle
$$

through an expansion over a set of fully antisymmetric $A$-body basis states including both discrete square-integrable wave functions and continuous binary-cluster channel states of angular momentum $J$, parity $\pi$ and isospin $T$. Here, $\left|A \lambda J^{\pi} T\right\rangle$ are antisymmetric many-body eigenstates with eigenvalue $E_{\lambda}^{J^{\pi} T}$ obtained by diagonalizing the microscopic $A$-nucleon Hamiltonian within the NCSM basis

$$
\hat{H}\left|A \lambda J^{\pi} T\right\rangle=E_{\lambda}\left|A \lambda J^{\pi} T\right\rangle
$$

whereas the continuous elements

$$
\left|\Phi_{\nu r}^{J^{\pi} T}\right\rangle=\left[\left(\left|A-a \alpha_{1} I_{1}^{\pi_{1}} T_{1}\right\rangle\left|a \alpha_{2} I_{2}^{\pi_{2}} T_{2}\right\rangle\right)^{(s T)} Y_{\ell}\left(\hat{r}_{A-a, a}\right)\right]^{\left(J^{\pi} T\right)} \frac{\delta\left(r-r_{A-a, a}\right)}{r r_{A-a, a}} .
$$

are a set of translational-invariant cluster basis states describing two nuclei (a target and a projectile composed of $A-a$ and $a \leq A$ nucleons, respectively) whose centers of mass are separated by the relative coordinate $\vec{r}_{A-a, a}$ and that are traveling in a ${ }^{2 s} \ell_{J}$ wave of relative motion (with $s$ the channel spin, and $\ell$ the relative momentum of the system). The antisymmetric wave functions $\left|A-a \alpha_{1} I_{1}^{\pi_{1}} T_{1}\right\rangle$ and $\left|a \alpha_{2} I_{2}^{\pi_{2}} T_{2}\right\rangle$ are eigenstates of the $(A-a)$ - and $a$ nucleon intrinsic Hamiltonians, respectively, as obtained within the NCSM approach [1] and are characterized by the 
spin-parity, isospin and energy labels $I_{i}^{\pi_{i}}, T_{i}$, and $\alpha_{i}$, respectively, where $i=1,2$. In our notation, all these quantum numbers are grouped into a cumulative index $\nu=\left\{A-a \alpha_{1} I_{1}^{\pi_{1}} T_{1} ; a \alpha_{2} I_{2}^{\pi_{2}} T_{2} ; s \ell\right\}$. The full antisymmetrization of this continuous sector of the basis is enforced by introducing the appropriate inter-cluster antisymmetrizer, schematically

$$
\hat{\mathcal{A}}_{\nu}=\sqrt{\frac{(A-a) ! a !}{A !}}\left(1+\sum_{P \neq i d}(-)^{p} P\right)
$$

where the sum runs over all possible permutations of nucleons $P$ different from the identical one that can be carried out between the two different clusters, and $p$ is the number of interchanges characterizing them. Finally, $c_{\lambda}^{J^{\pi} T}$ and $\gamma_{\nu}^{J^{\pi} T}(r)$ are respectively discrete and continuous linear variational amplitudes obtained as solutions of the following coupled equations,

$$
\left(\begin{array}{cc}
H_{N C S M} & \bar{h} \\
\bar{h} & \overline{\mathcal{H}}
\end{array}\right)\left(\begin{array}{c}
c \\
\chi
\end{array}\right)=E\left(\begin{array}{ll}
1 & \bar{g} \\
\bar{g} & 1
\end{array}\right)\left(\begin{array}{l}
c \\
\chi
\end{array}\right)
$$

The new amplitudes $\chi_{\nu}^{J^{\pi} T}(r)$ are relative wave functions in the NCSM/RGM sector of the basis when working with orthogonalized cluster channel states of the type [9]

$$
\sum_{\nu^{\prime}} \int d r^{\prime} r^{\prime 2} \mathcal{N}_{\nu \nu^{\prime}}^{-\frac{1}{2}}\left(r, r^{\prime}\right) \hat{\mathcal{A}}_{\nu^{\prime}}\left|\Phi_{\nu^{\prime} r^{\prime}}^{J^{\pi} T}\right\rangle
$$

where $\mathcal{N}_{\nu \nu^{\prime}}^{-\frac{1}{2}}\left(r, r^{\prime}\right)$ is the inverse square root of the NCSM/RGM overlap (or norm) kernel

$$
\mathcal{N}_{\nu^{\prime} \nu}^{J^{\pi} T}\left(r^{\prime}, r\right)=\left\langle\Phi_{\nu^{\prime} r^{\prime}}^{J^{\pi} T}\left|\hat{\mathcal{A}}_{\nu^{\prime}} \hat{\mathcal{A}}_{\nu}\right| \Phi_{\nu r}^{J^{\pi} T}\right\rangle
$$

and the original wave functions $\gamma_{\nu}^{J^{\pi} T}(r)$ of Eq. (1) are related to $\chi_{\nu^{\prime}}^{J^{\pi} T}\left(r^{\prime}\right)$ by the relationship

$$
\frac{\gamma_{\nu}(r)}{r}=\sum_{\nu^{\prime}} \int d r^{\prime} r^{\prime 2} \mathcal{N}_{\nu \nu^{\prime}}^{-\frac{1}{2}}\left(r, r^{\prime}\right) \frac{\chi_{\nu^{\prime}}\left(r^{\prime}\right)}{r^{\prime}}
$$

Because of this orthogonalization procedure both diagonal blocks in the NCSMC norm kernel $N$ are identities in their respective spaces

$$
N_{\nu r \nu^{\prime} r^{\prime}}^{\lambda \lambda^{\prime}}=\left(\begin{array}{cc}
\delta_{\lambda \lambda^{\prime}} & \bar{g}_{\lambda \nu^{\prime}}\left(r^{\prime}\right) \\
\bar{g}_{\lambda^{\prime} \nu}(r) & \delta_{\nu \nu^{\prime}} \frac{\delta\left(r-r^{\prime}\right)}{r r^{\prime}}
\end{array}\right)
$$

The off-diagonal elements represent the coupling between the two sectors of the basis and are given by the cluster form factors:

$$
\bar{g}_{\lambda \nu}(r)=\sum_{\nu^{\prime}} \int d r^{\prime} r^{\prime 2}\left\langle A \lambda J^{\pi} T \mid \hat{\mathcal{A}}_{\nu^{\prime}} \Phi_{\nu^{\prime} r^{\prime}}^{J^{\pi} T}\right\rangle \mathcal{N}_{\nu^{\prime} \nu}^{-\frac{1}{2}}\left(r^{\prime}, r\right) .
$$

In the NCSMC Hamiltonian kernel, the NCSM sector is given by a diagonal matrix of the NCSM eigenvalues $E_{\lambda}(2)$,

$$
\left(H_{N C S M}\right)_{\lambda \lambda^{\prime}}=\left\langle A \lambda J^{\pi} T|\hat{H}| A \lambda^{\prime} J^{\pi} T\right\rangle=E_{\lambda} \delta_{\lambda \lambda^{\prime}},
$$

while $\overline{\mathcal{H}}$ is the orthogonalized and hermitized NCSM/RGM Hamiltonian kernel given by

$$
\overline{\mathcal{H}}_{\nu \nu^{\prime}}\left(r, r^{\prime}\right)=\sum_{\mu \mu^{\prime}} \iint d y d y^{\prime} y^{2} y^{\prime 2} \mathcal{N}_{\nu \mu}^{-\frac{1}{2}}(r, y)\left\langle\Phi_{\mu y}^{J^{\pi} T}\left|\frac{1}{2}\left(\hat{\mathcal{A}}^{2} \hat{H}-\hat{H} \hat{\mathcal{A}}^{2}\right)\right| \Phi_{\mu^{\prime} y^{\prime}}^{J^{\pi} T}\right\rangle \mathcal{N}_{\mu^{\prime} \nu^{\prime}}^{-\frac{1}{2}}\left(y^{\prime}, r^{\prime}\right),
$$

and $\bar{h}_{\lambda \nu}(r)$ is the coupling form factor:

$$
\bar{h}_{\lambda \nu}(r)=\sum_{\nu^{\prime}} \int d r^{\prime} r^{\prime 2}\left\langle A \lambda J^{\pi} T\left|\hat{H} \hat{\mathcal{A}}_{\nu^{\prime}}\right| \Phi_{\nu^{\prime} r^{\prime}}^{J^{\pi} T}\right\rangle \mathcal{N}_{\nu^{\prime} \nu}^{-\frac{1}{2}}\left(r^{\prime}, r\right)
$$

We refer the interested reader to Refs. [9] and [12] for more details on the NCSM/RGM integration kernels. Algebraic expressions for the cluster form factor $\bar{g}_{\lambda \nu}(r)$ and the coupling form factor $\bar{h}_{\lambda \nu}(r)$ can be found in Ref. [15]. The 
calculation of $\left\langle A \lambda J^{\pi} T \mid \hat{\mathcal{A}}_{\nu} \Phi_{\nu r}^{J^{\pi} T}\right\rangle$ matrix elements with NCSM wave functions was already discussed in Ref. [17]. We also note that by squaring the absolute value of these matrix elements and integrating over $r$, one obtains spectroscopic factors.

The NCSMC equations can be orthogonalized in an analogous way to that presented for the NCSM/RGM in Ref. [9]. More specifically, when computing the norm kernel, the "exchange" term arising from the permutations in $\hat{\mathcal{A}}_{\nu}$ that differ from the identity are obtained by expanding the radial dependence of the basis states of Eq. (3) on HO radial wave functions $R_{n \ell}(r)$. This $\mathrm{HO}$ basis has the same frequency used in the NCSM cluster calculations and its size is consistent with the model space used in the cluster diagonalizations. To define the square and inverse square root of the NCSMC norm in the $r$-space representation, we first rewrite Eq. (9) as the convolution of the model-space norm kernel plus a correction for the finite size of the $\mathrm{HO}$ model-space $P$

$$
N_{\nu r \nu^{\prime} r^{\prime}}^{\lambda \lambda^{\prime}}=\left(\begin{array}{cc}
0 & 0 \\
0 & \delta_{\nu \nu^{\prime}} \frac{\delta\left(r-r^{\prime}\right)}{r r^{\prime}}-R_{n \ell}(r) \delta_{\nu \nu^{\prime}} \delta_{n n^{\prime}} R_{n^{\prime} \ell^{\prime}}\left(r^{\prime}\right)
\end{array}\right)+\left(\begin{array}{cc}
\delta_{\lambda \tilde{\lambda}} & 0 \\
0 & R_{\nu r \tilde{\nu} n}
\end{array}\right) N_{\tilde{\nu} n \tilde{\nu}^{\prime} n^{\prime}}\left(\begin{array}{cc}
\delta_{\tilde{\lambda}^{\prime} \lambda^{\prime}} & 0 \\
0 & R_{\nu^{\prime} r^{\prime} \tilde{\nu}^{\prime} n^{\prime}}^{\prime}
\end{array}\right)
$$

with the model-space NCSMC norm given by

$$
N_{\tilde{\nu} n \tilde{\nu}^{\prime} n^{\prime}}^{\tilde{\lambda} \tilde{\lambda}^{\prime}}=\left(\begin{array}{cc}
\delta_{\tilde{\lambda} \tilde{\lambda}^{\prime}} & \bar{g}_{\tilde{\lambda} \tilde{\nu}^{\prime} n^{\prime}} \\
\bar{g}_{\tilde{\lambda}^{\prime} \tilde{\nu} n} & \delta_{\tilde{\nu} \tilde{\nu}^{\prime}} \delta_{n n^{\prime}}
\end{array}\right)
$$

Here, $R_{\nu r \tilde{\nu} n}=R_{n \ell}(r) \delta_{\nu \tilde{\nu}}$, the model-space cluster form factor is related to the r-space one through $\bar{g}_{\lambda \nu}(r)=$ $\sum_{n} R_{n l}(r) \bar{g}_{\lambda \nu n}$, and the sum over the repeating indexes $\tilde{\lambda}, \tilde{\nu}, n, \tilde{\lambda}^{\prime}, \tilde{\nu}^{\prime}$, and $n^{\prime}$ is implied. The square and inverse square roots of $N$ can then be defined as:

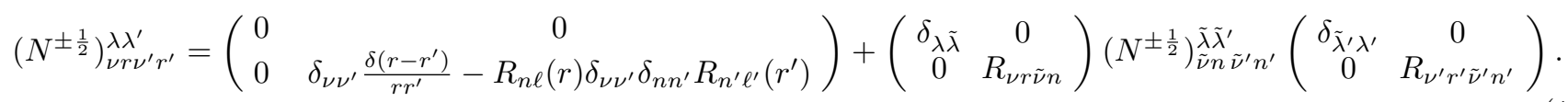

Inserting the identity $N^{-\frac{1}{2}} N^{+\frac{1}{2}}$ in both left- and right-hand sides of Eq. (5), and multiplying by $N^{-\frac{1}{2}}$ from the left, one obtains,

$$
\bar{H}\left(\begin{array}{l}
\bar{c} \\
\bar{\chi}
\end{array}\right)=E\left(\begin{array}{l}
\bar{c} \\
\bar{\chi}
\end{array}\right),
$$

where the orthogonalized NCSMC Hamiltonian $\bar{H}$ is given by,

$$
\bar{H}=N^{-\frac{1}{2}}\left(\begin{array}{cc}
H_{N C S M} & \bar{h} \\
\bar{h} & \overline{\mathcal{H}}
\end{array}\right) N^{-\frac{1}{2}},
$$

and the orthogonal wave functions by:

$$
\left(\begin{array}{l}
\bar{c} \\
\bar{\chi}
\end{array}\right)=N^{+\frac{1}{2}}\left(\begin{array}{l}
c \\
\chi
\end{array}\right) .
$$

Starting from (1), the orthogonalized NCSMC wave function takes then the form:

$$
\begin{aligned}
\left|\Psi_{A}^{J^{\pi} T}\right\rangle & =\sum_{\lambda}\left|A \lambda J^{\pi} T\right\rangle\left[\sum_{\lambda^{\prime}}\left(N^{-\frac{1}{2}}\right)^{\lambda \lambda^{\prime}} \bar{c}_{\lambda^{\prime}}+\sum_{\nu^{\prime}} \int d r^{\prime} r^{2}\left(N^{-\frac{1}{2}}\right)_{\nu^{\prime} r^{\prime}}^{\lambda} \frac{\bar{\chi}_{\nu^{\prime}}\left(r^{\prime}\right)}{r^{\prime}}\right] \\
& +\sum_{\nu \nu^{\prime}} \int d r r^{2} \int d r^{\prime} r^{\prime 2} \hat{\mathcal{A}}_{\nu}\left|\Phi_{\nu r}^{J^{\pi} T}\right\rangle \mathcal{N}_{\nu \nu^{\prime}}^{-\frac{1}{2}}\left(r, r^{\prime}\right)\left[\sum_{\lambda^{\prime}}\left(N^{-\frac{1}{2}}\right)_{\nu^{\prime} r^{\prime}}^{\lambda^{\prime}} \bar{c}_{\lambda^{\prime}}+\sum_{\nu^{\prime \prime}} \int d r^{\prime \prime} r^{\prime \prime 2}\left(N^{-\frac{1}{2}}\right)_{\nu^{\prime} r^{\prime} \nu^{\prime \prime} r^{\prime \prime}} \frac{\bar{\chi}_{\nu^{\prime \prime}}\left(r^{\prime \prime}\right)}{r^{\prime \prime}}\right] .
\end{aligned}
$$

\section{A. Solving the NCSMC equations}

At large inter-cluster distances $r$, the clusters are assumed to interact through the Coulomb interaction only. Hence, the NCSMC equations are solved dividing the space into an internal region $r \leqslant a$ and an external region $r>a$ and applying the coupled-channel microscopic R-matrix method on a Lagrange mesh [18]. The separation radius $r=a$ 
must be large enough to ensure that the wave function of the $A$-body states $\left|A \lambda J^{\pi} T\right\rangle$ vanishes when approaching the external region, where the asymptotic behavior of the NCSMC solutions is described by the radial wave functions

$$
u_{\nu}^{J^{\pi} T}(r)=C_{\nu}^{J^{\pi} T} W_{l}\left(\eta_{\nu}, \kappa_{\nu} r\right)
$$

and

$$
u_{\nu}^{J^{\pi} T}(r)=\frac{i}{2} v_{\nu}^{-\frac{1}{2}}\left[\delta_{\nu i} H_{l}^{-}\left(\eta_{\nu}, \kappa_{\nu} r\right)-S_{\nu i}^{J^{\pi} T} H_{l}^{+}\left(\eta_{\nu}, \kappa_{\nu} r\right)\right]
$$

for bound and scattering states, respectively. Here, $W_{l}\left(\eta_{\nu}, \kappa_{\nu} r\right)$ are Wittaker functions and $H_{l}^{ \pm}\left(\eta_{\nu}, \kappa_{\nu} r\right)$ are the incoming and outgoing Coulomb functions. The scattering states are defined through the scattering matrix $S_{\nu i}^{J^{\pi} T}$ between the initial channel $i$ and final channel $\nu$. The function $u_{\nu}^{J^{\pi} T}(r)$ stands for either the non-orthogonalized wave function $\chi_{\nu}^{J^{\pi} T}(r)$ or for the orthogonalized $\bar{\chi}_{\nu}^{J^{\pi} T}(r)$ (Eqs. (17), (19), (20)).

One of the advantages of the microscopic R-matrix method is that the wave function $\bar{\chi}_{\nu}^{J^{\pi} T}(r)$ in the internal region can be expanded on a set of square-integrable functions. We adopted here the set of Lagrange functions $f_{\underline{n}}(r)$ associated with the shifted Lagrange polynomials and defined on the mesh points $r_{\underline{n}} \in[0, a][18]$. When the GaussLegendre quadrature approximation is adopted, the Lagrange functions are orthogonal to each other. Here, the index $\underline{n}$ labels the mesh points, whose number has to be large enough to have a correct representation of the wave functions.

The matching between internal and external regions, and hence the imposition of the asymptotic behavior of Eqs. (21) and/or (22), is ensured by the Bloch surface operator

$$
\hat{L}_{\nu}=\left(\begin{array}{cc}
0 & 0 \\
0 & \frac{1}{2} \delta(r-a)\left(\frac{d}{d r}-\frac{B_{\nu}}{r}\right)
\end{array}\right)
$$

and solving the Bloch-Schrödinger equations

$$
(\hat{\bar{H}}+\hat{L}-E)\left(\begin{array}{c}
\bar{c} \\
\bar{\chi}
\end{array}\right)=\hat{L}\left(\begin{array}{c}
\bar{c} \\
\bar{\chi}
\end{array}\right) .
$$

$\hat{\bar{H}}+\hat{L}$ is Hermitian when the boundary parameter $B_{\nu}$ is real. Because of the Bloch operator, the wave function in the right hand side (r.h.s.) of Eq. (24) can be replaced by its asymptotic behavior. When searching for bound states, $B_{\nu}$ is chosen in such a way that the r.h.s. vanishes, and one is left with the diagonalization problem:

$$
(\hat{\bar{H}}+\hat{L})\left(\begin{array}{c}
\bar{c} \\
\bar{\chi}
\end{array}\right)=E\left(\begin{array}{c}
\bar{c} \\
\bar{\chi}
\end{array}\right) .
$$

For scattering states, the scattering matrix $S$ and the wave functions are computed by solving Eq. (24) with the boundary parameter $B_{\nu}=0$ for each value of the relative kinetic energy $E_{k i n}$ of the projectile-target system. The phase shifts $\delta\left(E_{k i n}\right)$ can then be extracted from the S-matrix. Energetically open and closed channels are treated on equal footing when applying the microscopic R-matrix method.

\section{REDUCED MATRIX ELEMENTS OF THE E1 OPERATOR}

The electric dipole operator we use in the following is given by:

$$
\overrightarrow{E 1}=e \sum_{i=1}^{A} \frac{1+\tau_{i}^{(3)}}{2}\left(\vec{r}_{i}-\vec{R}_{\mathrm{c} . \mathrm{m} .}^{(A)}\right)
$$

where $e$ is the electric charge, $\vec{r}_{i}$ and $\tau_{i}$ the position vector and isospin of the ith nucleon, and

$$
\vec{R}_{\mathrm{c} . \mathrm{m} .}^{(A)}=\frac{1}{A} \sum_{i=1}^{A} \vec{r}_{i}
$$

the center of mass (c.m.) of the $A$-nucleon system. Working within a binary cluster basis, it is convenient to re-write Eq. (26) in terms of three components, an operator acting exclusively on the first $A-a$ nucleons (pertaining to 
the first cluster or target), an operator acting exclusively on the last a nucleons (belonging to the second cluster or projectile), and, finally, an operator acting on the relative motion wave function between target and projectile:

$$
\vec{E} 1=e \sum_{i=1}^{A-a} \frac{1+\tau_{i}^{(3)}}{2}\left(\vec{r}_{i}-\vec{R}_{\mathrm{c} . \mathrm{m} .}^{(A-a)}\right)+e \sum_{j=A-a+1}^{A} \frac{1+\tau_{j}^{(3)}}{2}\left(\vec{r}_{i}-\vec{R}_{\mathrm{c} . \mathrm{m} .}^{(a)}\right)+e \frac{Z_{(A-a)} a-Z_{(a)}(A-a)}{A} \vec{r}_{A-a, a} .
$$

Here, $\vec{R}_{\text {c.m. }}^{(A-a)}$ and $\vec{R}_{\text {c.m. }}^{(a)}$ are the centers of mass of the $(A-a)$ - and $a$-nucleon systems, respectively, while $Z_{(A-a)}$ and $Z_{(a)}$ represent respectively the charge numbers of the target and of the projectile. It can be easily demonstrated that Eqs. (26) and (28) are exactly equivalent.

The reduced matrix element of the E1 dipole operator between two bound states of an $A$-body nucleus with spin $J_{i}$, parity $\pi_{i}$, isospin $T_{i}$, energy $E_{i}$ in the initial state and $J_{f}, \pi_{f}, T_{f}, E_{f}$ in the final state is given by:

$$
\begin{aligned}
M_{f i}^{E 1} & =\left\langle\Psi_{A}^{J_{f}^{\pi_{f}} T_{f}}\left(E_{f}\right)\|E 1\| \Psi_{A}^{J_{i}^{\pi_{i}} T_{i}}\left(E_{i}\right)\right\rangle \\
& =\frac{\sqrt{2 J_{f}+1}}{C_{J_{i} M_{i} 1 \mu}^{J_{f} M_{f}}}\left\langle\Psi_{A}^{f M_{f}}\left(E_{f}\right)\left\|\sqrt{\frac{4 \pi}{3}} e \sum_{i=1}^{A} \frac{1+\tau_{i}^{(3)}}{2}\left|\vec{r}_{i}-\vec{R}_{\mathrm{c} . \mathrm{m} .}^{(A)}\right| Y_{1 \mu}\left(\widehat{\left.r_{i}-R_{\mathrm{c} . \mathrm{m} .}^{(A)}\right)}\right)\right\| \Psi_{A}^{i M_{i}}\left(E_{i}\right)\right\rangle .
\end{aligned}
$$

In the second line of Eq. (29) we have introduced the Clebsh-Gordan coefficient $C_{J_{i} M_{i} 1 \mu}^{J_{f} M_{f}}$ and the short notation $f(i)$ for the group of quantum numbers $\left\{J_{f(i)}^{\pi_{f(i)}} T_{f(i)}\right\}$ that will be used throughout the rest of this report.

In the NCSMC formalism the matrix element of Eq. (29) is given by the sum of four components, and specifically, the reduced matrix element in the NCSM sector of the wave function, the "coupling" reduced matrix elements between NCSM and NCSM/RGM (and vice versa) basis states, and the reduced matrix element in the NCSM/RGM sector:

$$
\begin{aligned}
M_{f i}^{E 1}= & \sum_{\lambda \lambda^{\prime}} c_{\lambda^{\prime}}^{f}\left\langle A \lambda^{\prime} J_{f}^{\pi_{f}} T_{f}\|E 1\| A \lambda J_{i}^{\pi_{i}} T_{i}\right\rangle c_{\lambda}^{i} \\
& +\sum_{\lambda^{\prime} \nu} \int d r r^{2} c_{\lambda^{\prime}}^{f}\left\langle A \lambda^{\prime} J_{f}^{\pi_{f}} T_{f}\left\|E 1 \hat{\mathcal{A}}_{\nu}\right\| \Phi_{\nu r}^{i}\right\rangle \frac{\gamma_{\nu}^{i}(r)}{r} \\
& +\sum_{\lambda \nu^{\prime}} \int d r^{\prime} r^{\prime 2} \frac{\gamma_{\nu^{\prime}}^{f}\left(r^{\prime}\right)}{r^{\prime}}\left\langle\Phi_{\nu^{\prime} r^{\prime}}^{f}\left\|\hat{\mathcal{A}}_{\nu^{\prime}} E 1\right\| A \lambda J_{i}^{\pi_{i}} T_{i}\right\rangle c_{\lambda}^{i} \\
& +\sum_{\nu \nu^{\prime}} \int d r^{\prime} r^{\prime 2} \int d r r^{2} \frac{\gamma_{\nu^{\prime}}^{f}\left(r^{\prime}\right)}{r^{\prime}}\left\langle\Phi_{\nu^{\prime} r^{\prime}}^{f}\left\|\hat{\mathcal{A}}_{\nu^{\prime}} E 1 \hat{\mathcal{A}}_{\nu}\right\| \Phi_{\nu r}^{i}\right\rangle \frac{\gamma_{\nu}^{i}(r)}{r} .
\end{aligned}
$$

The algebraic expression for the reduced matrix elements in the NCSM sector $\left\langle A \lambda^{\prime} J_{f}^{\pi_{f}} T_{f}\|E 1\| A \lambda J_{i}^{\pi_{i}} T_{i}\right\rangle$ can be easily obtained working in the single-particle Slater-Determinant (SD) harmonic oscillator basis and adopting the E1 operator in the form of Eq. (26). In the following, we consider the reduced matrix elements in the NCSM/RGM sector. First, we notice that the inter-cluster antisymmetrizer commutes with the $A$-nucleon $E 1$ dipole operator of Eq. (26) and

$$
\left\langle\Phi_{\nu^{\prime} r^{\prime}}^{f}\left\|\hat{\mathcal{A}}_{\nu^{\prime}} E 1 \hat{\mathcal{A}}_{\nu}\right\| \Phi_{\nu r}^{i}\right\rangle=\frac{1}{2}\left(\left\langle\Phi_{\nu^{\prime} r^{\prime}}^{f}\left\|\hat{\mathcal{A}}_{\nu^{\prime}} \hat{\mathcal{A}}_{\nu} E 1\right\| \Phi_{\nu r}^{i}\right\rangle+\left\langle\Phi_{\nu^{\prime} r^{\prime}}^{f}\left\|E 1 \hat{\mathcal{A}}_{\nu^{\prime}} \hat{\mathcal{A}}_{\nu}\right\| \Phi_{\nu r}^{i}\right\rangle\right) .
$$

Second, using the $E 1$ operator in the form of Eq. (28) we can rewrite, e.g., the first matrix element in the right-hand side of Eq. (31) as:

$$
\begin{aligned}
\left\langle\Phi_{\nu^{\prime} r^{\prime}}^{f}\left\|\hat{\mathcal{A}}_{\nu^{\prime}} \hat{\mathcal{A}}_{\nu} E 1\right\| \Phi_{\nu r}^{i}\right\rangle= & \left\langle\Phi_{\nu^{\prime} r^{\prime}}^{f}\left\|\hat{\mathcal{A}}_{\nu^{\prime}} \hat{\mathcal{A}}_{\nu} e \sum_{i=1}^{A-a} \frac{1+\tau_{i}^{(3)}}{2}\left(\vec{r}_{i}-\vec{R}_{\mathrm{c} . \mathrm{m} .}^{(A-a)}\right)\right\| \Phi_{\nu r}^{i}\right\rangle \\
& +\left\langle\Phi_{\nu^{\prime} r^{\prime}}^{f}\left\|\hat{\mathcal{A}}_{\nu^{\prime}} \hat{\mathcal{A}}_{\nu} e \sum_{j=A-a+1}^{A} \frac{1+\tau_{j}^{(3)}}{2}\left(\vec{r}_{j}-\vec{R}_{\mathrm{c} . \mathrm{m} .}^{(a)}\right)\right\| \Phi_{\nu r}^{i}\right\rangle \\
& +e \frac{Z_{(A-a)} a-Z_{(a)}(A-a)}{A}\left\langle\Phi_{\nu^{\prime} r^{\prime}}^{f}\left\|\hat{\mathcal{A}}_{\nu^{\prime}} \hat{\mathcal{A}}_{\nu} \vec{r}_{A-a, a}\right\| \Phi_{\nu r}^{i}\right\rangle
\end{aligned}
$$

If there are no allowed E1 transitions between the target (and projectile) eigenstates in the initial state and those in the final state (e.g., there are only positive-parity eigenstates of the target/projectile), the first two terms on the right 
hand side of Eq. (32) are equal to zero and one obtains:

$$
\begin{aligned}
\left\langle\Phi_{\nu^{\prime} r^{\prime}}^{f}\left\|\hat{\mathcal{A}}_{\nu^{\prime}} \hat{\mathcal{A}}_{\nu} E 1\right\| \Phi_{\nu r}^{i}\right\rangle= & e \frac{Z_{(A-a)} a-Z_{(a)}(A-a)}{A}\left\langle\Phi_{\nu^{\prime} r^{\prime}}^{f}\left\|\hat{\mathcal{A}}_{\nu^{\prime}} \hat{\mathcal{A}}_{\nu} \vec{r}_{A-a, a}\right\| \Phi_{\nu r}^{i}\right\rangle \\
= & e \frac{Z_{(A-a)} a-Z_{(a)}(A-a)}{A} \sqrt{\left(2 J_{i}+1\right)\left(2 J_{f}+1\right)(2 \ell+1)(-)^{s+J_{f}}} \delta_{T_{i} T_{f}} \\
& \times \sum_{\tilde{\ell}} \sqrt{2 \tilde{\ell}+1}\left(\begin{array}{ccc}
1 & \ell & \tilde{\ell} \\
0 & 0 & 0
\end{array}\right)\left\{\begin{array}{ccc}
\ell & s & J_{i} \\
J_{f} & 1 & \tilde{\ell}
\end{array}\right\} \mathcal{N}_{\nu^{\prime} \tilde{\nu}}^{f}\left(r^{\prime}, r\right) r
\end{aligned}
$$

where all quantum numbers in the index $\tilde{\nu}$ are identical to those in the index $\nu$ except for the angular momentum $\ell$, which is replaced by $\tilde{\ell}$.

The "coupling" E1 reduced matrix element between NCSM and NCSM/RGM components of the basis can be derived making similar considerations:

$$
\begin{aligned}
\left\langle A \lambda^{\prime} J_{f}^{\pi_{f}} T_{f}\left\|E 1 \hat{\mathcal{A}}_{\nu}\right\| \Phi_{\nu r}^{i}\right\rangle= & \left\langle A \lambda^{\prime} J_{f}^{\pi_{f}} T_{f}\left\|\hat{\mathcal{A}}_{\nu} E 1\right\| \Phi_{\nu r}^{i}\right\rangle \\
= & \left\langle A \lambda^{\prime} J_{f}^{\pi_{f}} T_{f}\left\|\hat{\mathcal{A}}_{\nu} e \sum_{i=1}^{A-a} \frac{1+\tau_{i}^{(3)}}{2}\left(\vec{r}_{i}-\vec{R}_{\mathrm{c} . \mathrm{m} .}^{(A-a)}\right)\right\| \Phi_{\nu r}^{i}\right\rangle \\
& +\left\langle A \lambda^{\prime} J_{f}^{\pi_{f}} T_{f}\left\|\hat{\mathcal{A}}_{\nu} e \sum_{j=A-a+1}^{A} \frac{1+\tau_{j}^{(3)}}{2}\left(\vec{r}_{j}-\vec{R}_{\mathrm{c} . \mathrm{m} .}^{(a)}\right)\right\| \Phi_{\nu r}^{i}\right\rangle \\
& +e \frac{Z_{(A-a)} a-Z_{(a)}(A-a)}{A}\left\langle A \lambda^{\prime} J_{f}^{\pi_{f}} T_{f}\left\|\hat{\mathcal{A}}_{\nu} \vec{r}_{A-a, a}\right\| \Phi_{\nu r}^{i}\right\rangle .
\end{aligned}
$$

Once again, the first two terms in the right-hand side of Eq. (34) can be disregarded provided that the internal excitations of the $(A-a)$-nucleon target (and those of the $a$-nucleon projectile) by the electric dipole operator have a negligible overlap with the $\left|A \lambda^{\prime} J_{f}^{\pi_{f}} T_{f}\right\rangle$ eigenstate. In such a case, one can make the approximation:

$$
\begin{aligned}
\left\langle A \lambda^{\prime} J_{f}^{\pi_{f}} T_{f}\left\|E 1 \hat{\mathcal{A}}_{\nu}\right\| \Phi_{\nu r}^{i}\right\rangle & =\frac{Z_{(A-a)} a-Z_{(a)}(A-a)}{A}\left\langle A \lambda^{\prime} J_{f}^{\pi_{f}} T_{f}\left\|\hat{\mathcal{A}}_{\nu} \vec{r}_{A-a, a}\right\| \Phi_{\nu r}^{i}\right\rangle \\
= & e \frac{Z_{(A-a)} a-Z_{(a)}(A-a)}{A} \sqrt{\left(2 J_{i}+1\right)\left(2 J_{f}+1\right)(2 \ell+1)(-)^{s+J_{f}}} \delta_{T_{i} T_{f}} \\
& \times \sum_{\tilde{\ell}} \sqrt{2 \tilde{\ell}+1}\left(\begin{array}{ccc}
1 & \ell & \tilde{\ell} \\
0 & 0 & 0
\end{array}\right)\left\{\begin{array}{ccc}
\ell & s & J_{i} \\
J_{f} & 1 & \tilde{\ell}
\end{array}\right\} g_{\lambda^{\prime} \nu}^{f}(r) r,
\end{aligned}
$$

where $g_{\lambda^{\prime} \nu}^{f}(r)=\left\langle A \lambda^{\prime} J_{f}^{\pi_{f}} T_{f} \mid \hat{\mathcal{A}}_{\nu} \Phi_{\nu r}^{J^{\pi} T}\right\rangle$ is the non-ortogonalized cluster form factor, related to the form factor of Eq. (10) by the relationship

$$
g_{\lambda \nu}(r)=\sum_{\nu^{\prime}} \int d r^{\prime} r^{\prime 2} \bar{g}_{\lambda \nu^{\prime}}\left(r^{\prime}\right) \mathcal{N}_{\nu^{\prime} \nu}^{\frac{1}{2}}\left(r^{\prime}, r\right)
$$

and $\tilde{\nu}$ has the same meaning as in Eq. (33).

To summarize, in the following we provide the algebraic expressions for the reduced matrix elements of the E1 operator within the fully orthogonalized NCSMC basis, that is:

$$
\bar{M}_{f i}^{E 1}=\left(\begin{array}{ll}
\bar{c}^{f} & \bar{\chi}^{f}
\end{array}\right) N^{f-\frac{1}{2}}\left(\begin{array}{cc}
A^{f i} & B^{f i} \\
\bar{B}^{f i} & C^{f i}
\end{array}\right) N^{i-\frac{1}{2}}\left(\begin{array}{c}
\bar{c}^{i} \\
\bar{\chi}^{i}
\end{array}\right),
$$

where $\bar{c}^{i(f)}$ and $\bar{\chi}^{i(f)}$ are, respectively, the NCSM and NCSM/RGM components of the orthogonal NCSMC basis function of Eq. (20), $N^{f(i)^{-\frac{1}{2}}}$ the inverse-square roots of the NCSMC norm kernel, defined in Eq. (16), and $A^{f i}, B^{f i}, \bar{B}^{f i}$ 
and $C^{f i}$ four matrices given by:

$$
\begin{aligned}
A_{\lambda^{\prime} \lambda}^{f i} & =\left\langle A \lambda^{\prime} J_{f}^{\pi_{f}} T_{f}\|E 1\| A \lambda J_{i}^{\pi_{i}} T_{i}\right\rangle, \\
B_{\lambda^{\prime} \rho y}^{f i} & =\sum_{\tilde{\rho}} \int d \tilde{y} \tilde{y}^{2} \bar{g}_{\lambda^{\prime} \tilde{\rho}}^{f}(\tilde{y}) E_{\tilde{\rho} \rho}^{f i}(\tilde{y}, y) \\
\bar{B}_{\rho^{\prime} y^{\prime} \lambda}^{f i} & =\sum_{\tilde{\rho}^{\prime}} \int d \tilde{y}^{\prime} \tilde{y}^{\prime 2} \bar{E}_{\rho^{\prime} \tilde{\rho}^{\prime}}^{f i}\left(y^{\prime}, \tilde{y}^{\prime}\right) \bar{g}_{\lambda \tilde{\rho}^{\prime}}^{i}\left(\tilde{y}^{\prime}\right) \\
C_{\rho^{\prime} y^{\prime} \rho y}^{f i} & =\frac{1}{2}\left[E_{\rho^{\prime} \rho}^{f i}\left(y^{\prime}, y\right)+\bar{E}_{\rho^{\prime} \rho}^{f i}\left(y^{\prime}, y\right)\right] .
\end{aligned}
$$

Here, we have introduced the orthogonalized E1 integration kernel

$$
\begin{aligned}
E_{\rho^{\prime} \rho}^{f i}\left(y^{\prime}, y\right)= & \delta_{T_{i} T_{f}} \sqrt{\left(2 J_{i}+1\right)\left(2 J_{f}+1\right)} \sum_{\nu \tilde{\ell}} e \frac{Z_{(A-a)} a-Z_{(a)}(A-a)}{A} \\
& \times(-)^{s+J_{f}} \sqrt{(2 \ell+1)(2 \tilde{\ell}+1)}\left(\begin{array}{ccc}
1 & \ell & \tilde{\ell} \\
0 & 0 & 0
\end{array}\right)\left\{\begin{array}{ccc}
\ell & s & J_{i} \\
J_{f} & 1 & \tilde{\ell}
\end{array}\right\} \\
& \times \int d r r^{2} \mathcal{N}^{f} \underset{\rho^{\prime} \tilde{\nu}}{\frac{1}{2}}\left(y^{\prime}, r\right) r \mathcal{N}_{\nu \rho}^{i-\frac{1}{2}}(r, y),
\end{aligned}
$$

and its Hermitian conjugate

$$
\begin{aligned}
& \bar{E}_{\rho^{\prime} \rho}^{f i}\left(y^{\prime}, y\right)=(-)^{J_{f}-J_{i}} E_{\rho \rho^{\prime}}^{i f}\left(y, y^{\prime}\right) \\
& =\delta_{T_{i} T_{f}} \sqrt{\left(2 J_{i}+1\right)\left(2 J_{f}+1\right)} \sum_{\nu^{\prime} \tilde{\ell}^{\prime}} e \frac{Z_{\left(A-a^{\prime}\right)} a^{\prime}-Z_{\left(a^{\prime}\right)}\left(A-a^{\prime}\right)}{A} \\
& \times(-)^{s^{\prime}+J_{i}} \sqrt{\left(2 \ell^{\prime}+1\right)\left(2 \tilde{\ell}^{\prime}+1\right)}\left(\begin{array}{ccc}
1 & \ell^{\prime} & \tilde{\ell}^{\prime} \\
0 & 0 & 0
\end{array}\right)\left\{\begin{array}{ccc}
\ell^{\prime} & s^{\prime} & J_{f} \\
J_{i} & 1 & \tilde{\ell}^{\prime}
\end{array}\right\} \\
& \times \int d r^{\prime} r^{\prime 2} \mathcal{N}_{\rho^{\prime} \nu^{\prime}}^{-\frac{1}{2}}\left(y^{\prime}, r^{\prime}\right) r^{\prime} \mathcal{N}_{\tilde{\nu}^{\prime} \rho}^{i \frac{1}{2}}\left(r^{\prime}, y\right)
\end{aligned}
$$

where $a^{\prime}$ is the mass number of the projectile in the final state, the quantum numbers in the index $\tilde{\nu}^{\prime}$ are identical to those in the index $\nu^{\prime}$ except for $\ell^{\prime}$, which is replaced by $\tilde{\ell}^{\prime}$, and the phase $(-1)^{J_{f}-J_{i}}$ is a result of the symmetry properties of the reduced matrix elements of the E1 operator under Hermitian congiugation:

$$
\left\langle n^{\prime} J_{f}\|\overrightarrow{E 1}\| n J_{i}\right\rangle=(-1)^{J_{f}-J_{i}}\left\langle n J_{i}\left\|\overrightarrow{E 1^{\dagger}}\right\| n^{\prime} J_{f}\right\rangle^{*}
$$

\section{CONCLUSIONS AND OUTLOOK}

In conclusion, we reviewed a new unified approach to nuclear bound and continuum states based on the coupling of the no-core shell model with the no-core shell model/resonating group method, and provided the algebraic expressions for the reduced matrix elements of the electric dipole operator within such an extended formalism. This $a b$ initio method that we call no-core shell model with continuum leads to convergence properties superior to either NCSM or NCSM/RGM. The main feature is the coupling of the pre-diagonalized $A$-nucleon NCSM wave functions with the binary-cluster and possibly three-body-cluster NCSM/RGM wave functions with proper aymptotic boundary conditions. The NCSMC method has the capability to describe efficiently: $i$ ) short- and medium-range nucleonnucleon correlations thanks to the large HO basis expansions used to obtain the NCSM eigenstates, and 2) long-range cluster correlations thanks to the NCSM/RGM cluster basis expansion. The formalism presented in this technical report paves the way for the ab initio description of the enhanced E1 transitions from the ground state to the first excited state in ${ }^{9,11} \mathrm{Be}$, which provide among the most sensitive tests of modern realistic interactions and calculated many-body wave functions, and have been recently re-measured at TRIUMF using the low-energy Coulomb excitation technique to improve the experimental accuracy to within $5 \%$ [16]. 


\section{ACKNOWLEDGMENTS}

Prepared in part by LLNL under Contract DE-AC52-07NA27344. Support from the U. S. DOE/SC/NP (Work Proposal No. 59871), the Natural Sciences and Engineering Research Council of Canada (NSERC) Grant No. 4019452011, and from the U. S. Department of Energy Grant DE-FC02-07ER41457 is acknowledged. TRIUMF receives funding via a contribution through the National Research Council Canada. This research was supported in part by the PAI-P6-23 of the Belgian Office for Scientific Policy and by the European Unions Seventh Framework Programme under grant agreement No. 62010.

[1] P. Navrátil, J. P. Vary and B. R. Barrett, Phys. Rev. Lett. 84, 5728 (2000).

[2] K. Wildermuth and Y. C. Tang, A unified theory of the nucleus, (Vieweg, Braunschweig, 1977).

[3] Y. C. Tang, M. LeMere and D. R. Thompson, Phys. Rep. 47, 167 (1978).

[4] T. Fliessbach and H. Walliser, Nucl. Phys. A377, 84 (1982).

[5] K. Langanke and H. Friedrich, Advances in Nuclear Physics, edited by J. W. Negele and E. Vogt (Plenum, New York, 1986).

[6] R. G. Lovas, R. J. Liotta, A. Insolia, K. Varga and D. S. Delion, Phys. Rep. 294, 265 (1998).

[7] H. M. Hofmann and G. M. Hale, Phys. Rev. C 77, 044002 (2008).

[8] S. Quaglioni and P. Navrátil, Phys. Rev. Lett. 101, 092501 (2008).

[9] S. Quaglioni and P. Navrátil, Phys. Rev. C 79, 044606 (2009).

[10] P. Navrátil, R. Roth and S. Quaglioni, Phys. Rev. C 82, 034609 (2010).

[11] P. Navrátil, R. Roth, and S. Quaglioni, Physics Letters B 704, 379 (2011)

[12] P. Navrátil and S. Quaglioni, Phys. Rev. C 83, 044609 (2011).

[13] P. Navrátil and S. Quaglioni, Phys. Rev. Lett. 108, 042503 (2012).

[14] P. Navrátil, S. Quaglioni, I. Stetcu and B. R. Barrett, J. Phys. G 36, 083101 (2009).

[15] S. Baroni, P. Navrátil, and S. Quaglioni, in preparation.

[16] E. Kwan, TIGRESS/BAMBINO collaboration, Bulletin of the American Physical Society, 2012 Fall Meeting of the APS Division of Nuclear Physics, Volume 57, Number 9, Abstract CD.00006.

[17] P. Navrátil, Phys. Rev. C 70, 054324 (2004).

[18] M. Hesse, J.-M. Sparenberg, F. Van Raemdonck, and D. Baye, Nucl. Phys. A640, 37 (1998); M. Hesse, J. Roland, and D. Baye, Nucl. Phys. A709, 184 (2002). 